and fast rules for doing so. In the above example, for instance, if we substitute some other number, $k$ say, for 2.507 , the desired effect does not occur for all values of $k$. An integral power of ten will not work (since we are assuming rounding-off is done in the decimal system). Also one finds experimentally in this case that $k=1.001$ and $k=2$ are unsatisfactory choices.

In our present study we might use a transformation of the type

$$
s=k s^{\prime}
$$

and re-evaluate the $\alpha_{j}$ in equation (2) by expanding the function

$$
\phi\left(s^{\prime}\right)=f\left(k s^{\prime}\right)
$$

around the point

$$
a^{\prime}=\frac{a}{k}
$$

obtaining quantities $\alpha_{j}^{\prime}$ as coefficients of $\left(s^{\prime}-a^{\prime}\right)^{i}$, from which, theoretically

$$
\alpha_{j}=\frac{\alpha_{j}^{\prime}}{k^{i}}
$$

Then by comparing the original quantities $\alpha_{j}$, with those obtained from (67), as $j$ increases, one could estimate the cumulative building up of round-off errors.

D. P. Flemming

Canadian Armament

Research and Development Establishment

P. O. Box 1427

Quebec, Que.

The main part of the work connected with the preparation of this paper was done while the author was employed at Minneapolis-Honeywell Regulator Company, Minneapolis, Minnesota.

1. C. K. Tirus, "A general card-program for the evaluation of the inverse Laplace transform," Assn. for Comp. Machinery, Journ., v. 2, 1955, p. 18-27.

2. H. S. CARSLAw \& J. C. JAEGER, Operational Methods in Applied Mathematics, Oxford Univ. Press, 2nd Edition, 1948.

3. WM. FELLER, An Introduction to Probability Theory and its Applications, John Wiley \& Sons, Inc., New York, 1950.

4. R. V. CHURCHILL, Introduction to Complex Variables and Applications, McGraw-Hill Book Co., Inc., New York, 1948.

\title{
Numerical Integration over Simplexes and Cones
}

1. Introduction. In this paper we develop numerical integration formulas for simplexes and cones in $n$-space for $n \geq 2$. While several papers have been written on numerical integration in higher spaces, most of these have dealt with hyperrectangular regions. For certain exceptions see [3]. Hammer and Wymore [1] have given a first general type theory designed through systematic use of cartesian product regions and affine transformations to extend the possible usefulness of formulas for each region. 
Particular formulas were developed by Hammer and Wymore for certain symmetrical type regions including spheres and hyper spheres, cubes and hypercubes. The methods they have used make it possible to obtain numerical integration formulas for a much larger class of regions than heretofore. However, it is not practical to obtain specific integration formulas for all regions of interest. Hence, in this paper we develop methods for obtaining numerical integration formulas over simplexes which may be used, in principle, to approximate other regions. Since one of the methods is applicable to cones in general if a formula is given for the base, we include that in the development.

While we have calculated certain formulas in specific cases for $n=2,3$, and 4, we use these as illustrations and hope to build a more complete table later. Inductively we give integration formulas holding exactly for the $k$-th degree polynomial in $n$ variables over the $n$-simplex. Another method which may be more valuable in some applications requires affine symmetry of the evaluation points. Here the general theory is missing, but a general type of method is proposed for which illustrations are provided.

2. Approximate integration formulas for cones. In the following a theorem of Hammer and Wymore will be used which we state as follows:

THEOREM 1. If

$$
\sum_{j} a_{j} f\left(\xi_{j}\right)-\int_{R} f(\xi) d V=E(f),
$$

then

$$
\sum_{j} W a_{j} g\left(\eta_{j}\right)-\int_{T R} g(\eta) d V=W E(f),
$$

where $T$ is an affine transformation, $\eta=A \xi+\eta_{0}$, of $E_{n}$ onto itself; $g(\eta)=f(\xi)$; $W$ is the absolute value of the determinant of $A ; R$ is an $n$-dimensional region included in the domain of $f$ and $\xi_{1}, \cdots, \xi_{k}, \cdots$, are points in the domain of $f$.

This theorem permits us to consider convenient, specific regions $R$ to develop formulas for the class of all their affine transforms.

We want integration formulas of the form

$$
\int_{R} f d V \doteq \sum_{1}^{k} a_{j} f\left(\xi_{j}\right),
$$

where the numbers $a_{j}$ are constants; $\xi_{1}, \cdots, \xi_{k}$ are points in the domain of $f ; R$ is a bounded closure of an open set in $E_{n}$. From this standpoint the most interesting simple regions are the simplexes, which are special cones, since every polyhedron is composed of a finite number of simplexes.

Let an $n$-dimensional region $R$ be embedded in the hyperplane $x=1$ in $E_{n+1}$ where we represent the points in $E_{n+1}$ by $(\xi, x)$, where $\xi$ is a point in $E_{n}$. Then the set of all points $x R$, where $0 \leq x \leq 1$, is a cone $C$ with base $R$ and vertex at the origin in $E_{n+1}$.

Let $f(\xi, x)$ be a function defined over $C$ and suppose that a suitable numerical integration formula is given over the base $R$ of $C$. If, for example,

$$
\int_{R} f(\xi, 1) d V_{n} \doteq \sum_{j} a_{j} f\left(\xi_{j}, 1\right)
$$


then

$$
\int_{C} f(\xi, x) d V=\int_{0}^{1} d x \int_{x R} f(\xi, x) d V_{n} \doteq \int_{0}^{1} x^{n} \sum_{j} a_{j} f\left(x \xi_{j}, x\right) d x,
$$

since the Jacobian of the affine transformation from $R$ to $x R$ is $x^{-n}$. Define a function

$$
g(x)=\sum_{j} a_{j} f\left(x \xi_{j}, x\right)
$$

and then we have

$$
\int_{C} f d v \doteq \int_{0}^{1} x^{n} g(x) d x
$$

Now we ask for numerical integration formulas of the form

$$
\int_{0}^{1} x^{n} g(x) d x \doteq \sum_{i} b_{i} g\left(x_{i}\right)
$$

Since such formulas may certainly be found we then have

$$
\int_{C} f d v \doteq \sum_{i} \sum_{j} b_{i} a_{j} f\left(x_{i} \xi_{j}, x_{i}\right)
$$

which is of the form required, that is, a weighted sum of integrand values.

THEOREM-2. If a formula (2) holds precisely for polynomials $f$ of $n$ variables of at most degree $m$ over a region $R$ and if a formula (4) holds precisely for polynomials $g$ of at most degree $m$ in $x$, then (5) holds over $C$ for all polynomial functions $f$ containing terms of at most degree $m$ in its $n+1$ variables.

We will forego a formal proof of Theorem 2 since it follows from the affine invariance of the class of polynomials of at most a certain degree, so that

$$
x^{n} \sum a_{j} f\left(x \xi_{j}, x\right)=\int_{x R} f(\xi, x) d V_{n}
$$

In order to develop specific formulas one may start with any of the numerous formulas for a line segment integration and proceed to obtain a formula for a triangle; using this obtain a formula for a tetrahedron and so on. This is the type we consider now. In higher spaces, to keep the number of points smaller, the integration formulas we choose for $\int_{0}^{1} x^{n} g(x) d x$ are based on orthogonal polynomials with weight function $x^{n}$ so that with $m$ values of $x_{j}$ the formula holds precisely for a polynomial of degree at most $2 m-1$ by formula (3). For $n=0$ this gives the Gauss integration formula on the line with points of evaluation the roots of the $m$-th Legendre polynomial. Then if the formula (2) for the base is exact for polynomials of at most degree $2 m-1$ in the first $n$ variables, the final formula holds for polynomials of degree $2 m-1$ at most. Thus by this means we obtain with $m^{n}$ points a formula valid for all $(2 m-1)$-degree polynomials over a simplex in $n$-space. While we have discussed a range from 0 to 1 , the affine invariance permits us to give the formulas as valid for all simplexes, the necessary 
adjustment of coefficients and points being given by the transformation as in Theorem 1.

The orthogonal polynomials, $q_{m}(x)$, over the interval $(0,1)$ with weight functions $x^{n}$ are given by Christoffel's formula in terms of the Legendre polynomials, $P_{m}(x)$, on the interval $(0,1)$ :

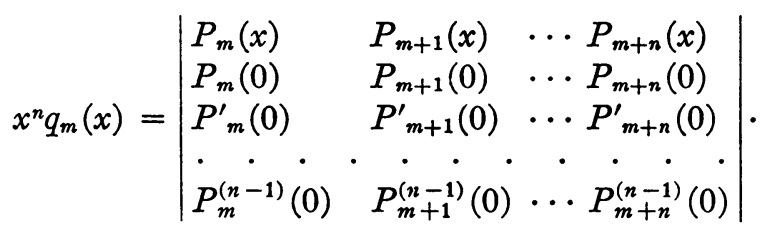

In this formula and in the tables the value of $n$ is one less than the dimension of the space-for the line, $n=0$; for the plane, $n=1$; and so on. The resulting

TABLE I. $n=1$
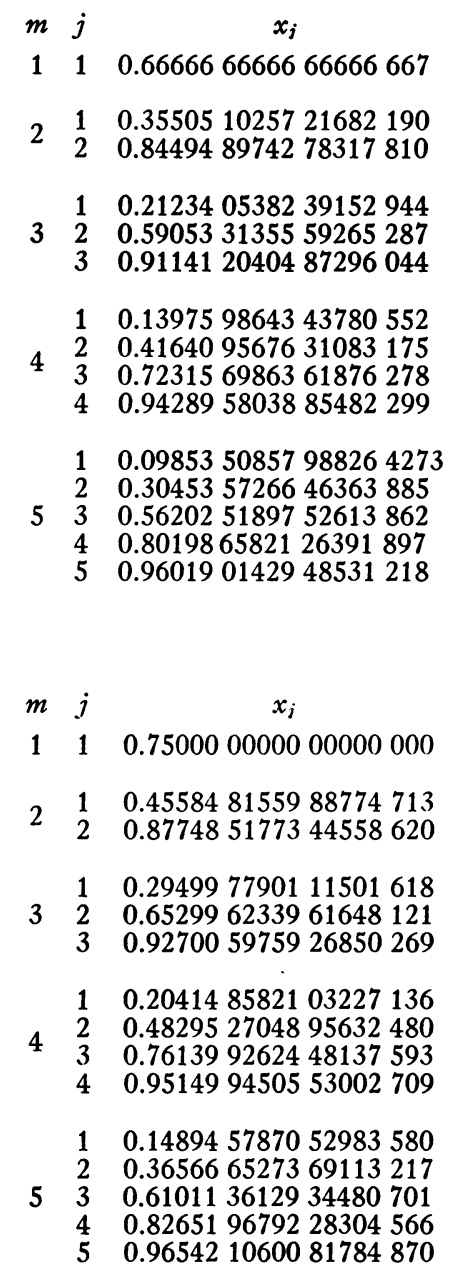$$
\text { TABLE II. } n=2
$$$$
b_{j}
$$

\subsection{0}

0.181958618256022831 0.318041381743977169

0.0698269799014541224

0.229241106359586248

0.200931913738959648

0.0311809709500080822

0.129847547608232439

0.203464568010271322

0.135506913431488149

0.0157479145216922766 0.0739088700726166584 0.146386987084669768 0.167174638094369395 0.0967815902266517818$$
0.333333333333333333
$$$$
0.100785882079825431
$$$$
0.232547451253507905
$$

0.0299507030085806981 0.146246269259866020 0.157136361064886615

0.0103522407499180652 0.0686338871729230663 0.143458789799214191 0.110888415611277894

0.00411382520309900782 0.0320556007229619169 0.0892001612215900168 0.126198961899911440 0.0817647842857709715

$$
\begin{gathered}
q_{m}(x) \\
\sqrt{4}(2-3 x) \\
\sqrt{6}\left(3-12 x+10 x^{2}\right) \\
\sqrt{8}\left(4-30 x+60 x^{2}-35 x^{3}\right) \\
\sqrt{10}\left(5-60 x+210 x^{2}-280 x^{3}\right. \\
\left.+126 x^{4}\right) \\
\sqrt{12}\left(6-105 x+560 x^{2}-1260 x^{3}\right. \\
\left.+1260 x^{4}-462 x^{5}\right)
\end{gathered}
$$


TABLE III. $n=3$

\begin{tabular}{ccclc}
$m$ & $j$ & $x_{i}$ & \multicolumn{1}{c}{$b_{i}$} & $g_{m}(x)$ \\
1 & 1 & 0.800000000000000000 & 0.250000000000000000 & $\sqrt{6}(4-5 x)$ \\
& & & & \\
2 & 1 & 0.529857935894884910 & 0.0669052498068887467 & $\sqrt{8}\left(10-30 x+21 x^{2}\right)$ \\
& 2 & 0.898713492676543662 & 0.183094750193111252 & \\
& 1 & 0.363264630216511947 & 0.0164790592826717230 & \\
3 & 2 & 0.698811269163613535 & 0.104599897556806681 & $\sqrt{10}\left(20-105 x+168 x^{2}-84 x^{3}\right)$ \\
& 3 & 0.937924100619874523 & 0.128921043160521608 & \\
& 1 & 0.261477788830889686 & 0.00465836706006948897 & \\
4 & 2 & 0.535846446088250229 & 0.0425417241427666674 & $\sqrt{12}\left(35-280 x+756 x^{2}-840 x^{3}\right.$ \\
4 & 3 & 0.790283229969286800 & 0.109004368938641000 & $\left.+330 x^{4}\right)$ \\
& 4 & 0.957847080566118662 & 0.0937955398585229295 &
\end{tabular}

TABLE IV. Integration Formula over Triangle Exact for 7 th Degree Polynomial

$\begin{array}{crc}k & y_{k} & a_{k} \\ 1 & 0.861136311594052580 & 0.347854845137453860 \\ 2 & 0.339981043584856264 & 0.652145154862546143 \\ 3 & -0.339981043584856264 & 0.652145154862546143 \\ 4 & -0.861136311594052580 & 0.347854845137453860\end{array}$

\begin{tabular}{ccccc}
\multicolumn{5}{c}{ Points of evaluation $\left(x_{j}, x_{j} y_{k}\right)$} \\
$j$ & $x_{j}$ & $x_{j} y_{1}=-x_{j} y_{4}$ & $x_{i} y_{2}=-x_{j} y_{3}$ \\
1 & 0.139759864343780552 & 0.120352294089888328 & 0.0475157045308764547 \\
2 & 0.416409567631083175 & 0.358585399182305152 & 0.141571359361934441 \\
3 & 0.723156986361876278 & 0.622736739939136723 & 0.245859666898990366 \\
4 & 0.942895803885482299 & 0.811961814775453378 & 0.320566699396768242
\end{tabular}

\begin{tabular}{ccc}
\multicolumn{4}{c}{$w_{j k}=b_{j} a_{k}=$ weights for points $\left(x_{j}, x_{j} y_{k}\right)$} \\
$j$ & $k=1,4$ & $k=2,3$ \\
1 & 0.0108464518210505090 & 0.0203345191289575733 \\
2 & 0.0451680985647398624 & 0.0846794490434925770 \\
3 & 0.0707761357961718794 & 0.132688432214099443 \\
4 & 0.0471367363867646765 & 0.0883701770447234729
\end{tabular}

$q_{m}(x)$ are orthogonal, but not necessarily normal. The roots are the values of $x_{j}$ required. Normalization of the $q_{m}(x)$ gives the weights $b_{j}$ by:

$$
b_{j}^{-1}=\sum_{i=0}^{m-1}\left[q_{i}\left(x_{j}\right)\right]^{2}
$$

where $x_{j}$ is a zero of $q_{m}(x)$.

The orthogonal polynomials, $q_{m}(x)$, over the interval $(0,1)$ with weight function $x^{n}$ are the Jacobi polynomials under the linear transformation $x^{\prime}=\frac{1}{2}(1+x)$. The standard definition of the Jacobi polynomials gives the weight function as $(1-x)^{\alpha}(1+x)^{\beta}$ and the interval of orthogonality as $(-1,1)$. In this case, $\alpha=0, \beta=n$ and the linear transformation given above takes $(1+x)^{n}$ into $\left(2 x^{\prime}\right)^{n}$ and $(-1,1)$ onto $(0,1)$. From this fact, explicit representations of $q_{m}(x)$ are available.

We consider an example of how to combine a formula for $n=0$ (the Gauss formula on the line) and one for $n=1$, to obtain a formula for the triangle. In the following discussion we consider a plane triangle with vertices $(0,0),(1,1)$ 
and $(1,-1)$. Use of the formulas may be made for arbitrary triangles by applying theorem 1. If we take, in each of the above cases, $m=4$, a formula is obtained which is exact for the general polynomial of degree $2 m-1=7$. For $n=1, x_{j}$ shall denote the roots of $q_{4}(x)$, and $b_{j}$ the corresponding weight. The roots of the Legendre polynomial, $P_{4}$, shall be denoted by $y_{k}$, and the corresponding weights by $a_{k}$. Table IV gives the values of $x_{j}, b_{j}$, and $y_{k}, a_{k}$; the points, $\left(x_{j}, x_{j} y_{k}\right)$, at which the integrand is to be evaluated, and the weights, $w_{j k}$, at these points.

The first three tables give: 1$)$ the polynomials $q_{m}(x)$ obtained from equation (6) for $n=1,2,3$ respectively, and values of $m$ indicated in each table, 2) the roots $x_{j}$ of $q_{m}(x)$, and 3 ) the values of $b_{j}$ obtained from equation (7). The calculations were made at the University of Wisconsin Numerical Analysis Laboratory using a CPC with Eugene Albright's 18-digit floating decimal board. The approximate error in the $x_{j}$ 's is no more than 1 in the seventeenth significant figure; the approximate error in the $b_{j}$ 's is no more than 1 in the sixteenth significant figure. The sum of the $b_{j}$ 's is $1 /(n+1)$ where $n$ is defined as in equation (6).

3. Symmetrical formulas. While the foregoing development makes it possible, in principle, to obtain numerical integration formulas for the $n$-simplex to hold exactly for polynomials of at most degree $k$, we do not mean to suggest that such formulas are the most desirable. One feature of these formulas is that they are unsymmetrical-i.e., in a given simplex the particular points of evaluation are not an invariant set under affine transformations taking the simplex onto itself.

In this section we give the preliminary results of our investigations resulting from a requirement of affine symmetry. We give these results since we believe that formulas for triangle and tetrahedron will be most useful and we have certain specific formulas for these regions.

The requirement of affine symmetry we make is simply this: If an integration formula involves calculation of the integrand at a certain point $P$ to be multiplied by a weight, $w$, then all images of $P$ under all affine transformations of the region onto itself will appear in the formula with the same weight, $w$. While such a requirement would appear to increase the number of points, in dealing with polynomials this is not the case; actually we have obtained fewer points than with the other method.

Let us represent points in the space as vectors and write the vertices of a triangle as $V_{1}, V_{2}, V_{3}$, and the centroid as $C=\frac{1}{3} \sum_{1}^{3} V_{i}$. The first affine invariant formula for the triangle is to use the centroid as the sole evaluation point with weight equal to the area. This method works for all bounded regions in all finite dimensional spaces to give a formula for the general linear function over the region. For simplexes or for hypersquares, this is likely to be useful.

The quadratic function over the triangle we have shown to be integrated exactly by evaluations at $r V_{i}+(1-r) C$, where $i=1,2,3$. Since this is an affinely symmetric set, we find that the weight (for each point) is one-third the area, and $r= \pm \frac{1}{2}$. For $r=+\frac{1}{2}$ the evaluation points are the distinct trisection points of the median chords and for $r=-\frac{1}{2}$ the evaluation points are the midpoints of the sides. These formulas we consider to offer prospects of extensive usefulness, especially the latter. Since the general quadratic function has 6 terms, we have used three fewer points than an arbitrary specification of evaluation points would have permitted. 
The cubic polynomial is integrated over the triangle with $r V_{i}+(1-r) C$ and $C$ as evaluation points where $r=\frac{2}{5}$; the weight associated with the centroid is $\left(-\frac{9}{16}\right) \Delta$ and the weight with each of the other three points is $(25 / 48) \Delta$, where $\Delta$ is the area of the triangle.

The quintic polynomial is integrated precisely with seven points in the triangle using $r V_{i}+(1-r) C$, weight $a ; s V_{i}+(1-s) C$, weight $b$; and $C$, weight $c$. We find $r=\frac{1+\sqrt{15}}{7}, s=\frac{1-\sqrt{15}}{7}$, and $a=\left(\frac{155-\sqrt{15}}{1200}\right) \Delta, b=\left(\frac{155+\sqrt{15}}{1200}\right) \Delta$, $c=(9 / 40) \Delta$. Since the general quintic polynomial in two variables has 21 terms this formula appears to be a type we call efficient noting that one might not hope to accomplish a formula with fewer than $7=21 / 3$ points. Here the " 3 " is the number of degrees of freedom for each point due to coordinates and weight. However, there are known hyperefficient formulas (cf. [1]), which use fewer points than indicated by this argument. While we will not reproduce the argument here, we used a triangle with vertices $(0,0),(1,-1),(1,1)$. Then the requirements of the affine symmetry of the formula with the form of the region assured that all monomials with odd powers of $y$ could be omitted. This left 12 equations. We chose five of these and solved them for $a, b, c, r$, and $s$, and verified that the remaining 7 were satisfied.

Over the tetrahedron we have a formula for the general quadratic in three variables involving $r V_{i}+(1-r) C, i=1,2,3,4$, and weight $a$. We calculate $r=1 / \sqrt{5}$ and the weight $a=\left(\frac{1}{4}\right) \Delta$, where $\Delta$ is the volume of the tetrahedron. Another formula with points outside the tetrahedron results if $r=-1 / \sqrt{5}$. This type of formula will generally be less used than one with points inside.

For the cubic polynomial over the tetrahedron we have $r=\frac{1}{3}, a=(9 / 20) \Delta$, and $c=\left(-\frac{4}{5}\right) \Delta$; the centroid $C$ must now be included and $c$ is its weight. This formula is efficient since it involves 5 points and the number of terms in the general cubic is 20 .

Generalization and extension of the affinely symmetric methods is now being carried out. However, these specific results seem sufficiently useful to include now.

4. Conclusion. In this paper we have taken a step towards obtaining reasonable numerical integration formulas over simplexes and cones. The cones which are not simplexes have not been emphasized. However, the method devised for cones permits obtaining a formula for integration over a cone (finite) provided a formula is at hand for the base. Thus later on we hope to obtain other formulas for the solid sphere which is a special cone based on its surface.

Error analysis has not been attempted. Experimental calculations on simple regions indicated that for "reasonable" functions there is a decisive factor in favor of the classical formulas here presented over Monte Carlo methods.

University of Wisconsin

P. C. Hammer

Madison, Wisconsin

Westinghouse Elec. Corp.

O. J. MARLOWE

Atomic Power Div.

Pittsburgh, Pennsylvania

Chemstrand Corp.

A. H. Stroud

Alabama 
This work is supported in part by a grant of Wisconsin Alumni Research Foundation funds made by the Graduate Research Committee.

1. P. C. HAMMER \& A. W. WyMORE, "Numerical integration over higher dimensional regions." Unpublished manuscript. 1939.

2. G. SzEGö, Orthogonal Polynomials, Am. Math. Soc. Colloguium Publications, v. 23, New York,

3. G. W. TyLER, "Numerical integration of functions of several variables," Canadian J. Math., v. 5,1953 , p. $393-412$.

\section{Numerical Integration over Simplexes}

1. Introduction. Integration formulae for numerical evaluation of integrals over the simplex in $n$-space have been given inductively by Hammer, Marlowe, and Stroud [1] so that it is possible in principle to determine a formula holding exactly for the $k$ th degree polynomial in $n$ variables. In the same paper certain affinely symmetric integration formulae are given for the triangle and tetrahedron. Using the theory proposed by Hammer and Wymore [2], it is possible to extend the usefulness of methods developed by transformations of the regions and by use of Cartesian products.

In this paper we give two integration formulae of affinely symmetric type for the simplex in $n$-space which respectively hold exactly for the quadratic polynomial and the cubic polynomial in $n$ variables. The method for establishing the exact values of integrals needed we believe is new in that the "numerical" formulae are used for the purpose.

2. The formula for cubic polynomials. Let the vertices of the $n$-simplex, $S_{n}$, be $V_{0}, \cdots, V_{n}$ and then its centroid is given by $C=\sum_{1}^{n} V_{i} /(n+1)$. Let $\Delta_{n}$ be the hypervolume of $S_{n}$.

THEOREM 1: An integration formula exact for the general cubic polynomial over $S_{n}$ for $n \geq 1$ is given by

$$
\int_{S_{n}} f d v_{n}=a_{n} \sum_{0}^{n} f\left(U_{i}\right)+c_{n} f(C)
$$

where

and

$$
a_{n}=\frac{(n+3)^{2}}{4(n+1)(n+2)} \Delta_{n} \quad c_{n}=\frac{-(n+1)^{2}}{4(n+2)} \Delta_{n}
$$

$$
U_{i}=\frac{2}{n+3} V_{i}+\frac{n+1}{n+3} C \quad i=0, \cdots, n .
$$

Proof: It may first be remarked that the points $U_{i}$ are on the median lines of $S_{n}$ and that the statement of the theorem is in symmetric form. In particular, under an affine transformation taking $S_{n}$ onto itself, the set of points $\left\{U_{i}\right\}$ is invariant and the centroid $C$ is fixed. Since there exists an affine transformation mapping any simplex in $E_{n}$ onto any other we may choose any particular simplex $S_{n}$ to carry out the proof. Our choice is specified by vertices as follows:

$$
\begin{array}{ll}
V_{0}=(0, \cdots, 0), & V_{1}=(1,0, \cdots, 0), \\
V_{2}=(1,1,0, \cdots, 0), \cdots, & V_{n}=(1,0, \cdots, 0,1) .
\end{array}
$$

